\title{
Rotating Machinery Library for Diagnosis
}

\author{
Tatsuro Ishibashi ${ }^{1}$ Bing Han ${ }^{2}$ Tadao Kawai ${ }^{3}$ \\ ${ }^{1}$ Meidensha Corporation, Japan, ishibashi-tat@mb . meidensha . co.jp \\ ${ }^{2,3}$ Department of Mechanical \& Physical Engineering, Osaka City University, Japan, \\ \{han, kawai\} @mech.eng.osaka-cu.ac.jp
}

\begin{abstract}
This paper presents our new rotating machinery library. Diagnosing the complex system accurately based on stochastic method requires an enormous amount of data, both with and without faults. Acquiring operation data with all kinds of faults for each components is very hard and costly. To generate data for rotating machinery diagnosis, we developed rotating machinery library using Modelica. It provides the basic components such as rotor, shaft, bearing, coupling, housing and support. Its component models are implemented on basis of rotor dynamics theory. This library makes it possible accessing rotating machinery operation data with various faults such as unbalanced rotor, shaft bending and ball bearing faults. To validate our models, we compared both Modelica simulation and experiment with a rotor kit as a test case.
\end{abstract}

\section{Keywords: Rotating Machinery, Vibration, Diagnosis}

\section{Introduction}

Preventive maintenance has been the main stay of industry for a long time. Recently, IoT (Internet of Things) and Industry4.0 have become very popular to manage and control a system such as manufacturing system. These have naturally increased the focus on Condition-Based Maintenance (CBM). IoT makes it possible monitoring the system state on time and accumulating a large amount of data. So many sensors and sensor network are attached to each component of a system for the purpose of data acquisition. By collecting and analyzing these acquired data, it makes feasible to manage a system efficiently or detect problem in a system with high accuracy at early stage.

Although this concept is very important, there are many difficulties in measurement. It is not always possible that we attach sensors where we would like to measure. Neither is it possible that we measure significant features of a system due to the lack of sensors.

By stochastic approach such as machine learning, fault detection of complex system accurately requires an enormous amount of data, both with and without faults to determine the threshold of signal amplitude. As a method of grasping the operating states with faults, it is conceivable to collect operation data by embedding the damaged part into the actual machine or continuing to operate it until it gets damaged. If the equipment is large, the lifetime is long, or the equipment is expensive, it is difficult to accumulate data. Rotating machinery equipment apply to the above. IoT will help us collect and acquire data. Still, accumulating data with all kinds of faults for each components is very hard and costly. It is desirable to analyze the state when faults occur in the rotating machinery. Some simple fault cases are schematized and modeled. It is possible to generate data of rotating machinery equipment with faults such as unbalanced rotor, shaft bending, ball bearing faults and misalignment of coupling by simulation.

Modelica is an object-oriented, declarative, multidomain modeling language for component-oriented modeling of complex systems. It is a powerful tool which simulates a complex physical system. Almost all design parameters such as shape and rigidity are easily set to a model and a variable behavior of a certain component in a system is easily obtained. By building Modelica cyber system, we can also obtain physical quantities and features of components which are inaccessible in a real physical system. A model-based diagnosis and design approach including fault mode with Modelica has recently reported (Klenk et al, 2014; Minhas et al, 2014).

Hence, we focus on developing rotating machinery library based on well-established rotor dynamics theory using Modelica. It provides the basic components such as rotor, shaft, bearing, coupling, housing and support. Our final goal is generation of training data including unmeasurable quantities for diagnosis by statistical classification algorithms.

To validate our models, we compared both Modelica simulation and experiment with a rotor kit as a test case. We calibrated the fault related parameter embedded in Modelica model so that simulation results were in good agreement with the experiment. Data acquisition from physical system was done through COMEDI (COntrol and MEasurement Device Interface for Linux / RTAI).

The followings show our new rotating machinery library, the example of generated data from this library and validation of this library with a rotor kit as a test case. 


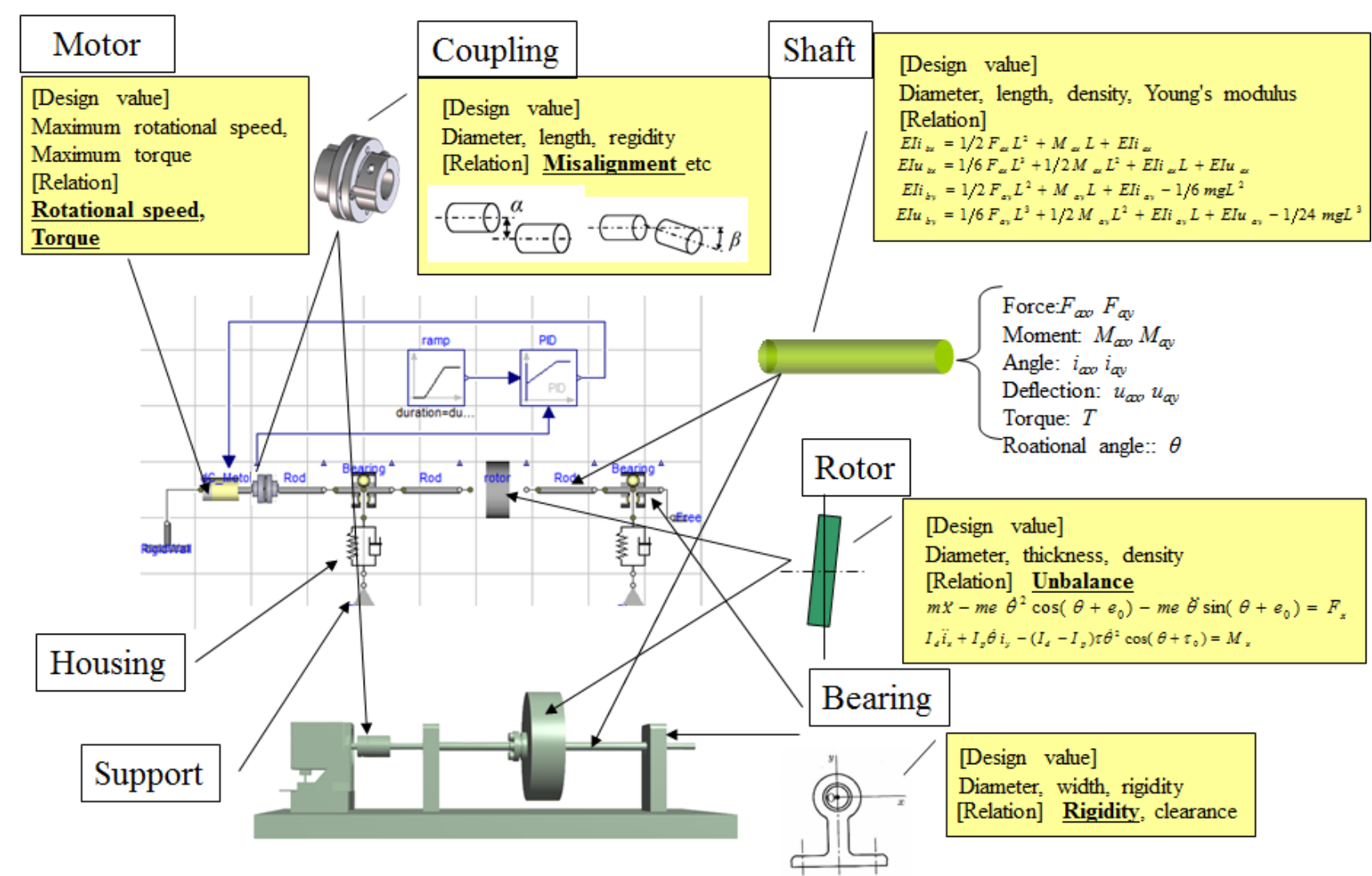

Figure 1. An example of a rotating machinery system. The upper figure describes our new library components of Modelica cyber system. The lower figure is corresponding physical system (The image is designed by CAD tool).

\section{Rotating Machinery Library}

Generally, a rotating machinery system has many problems. Static and dynamic unbalance of a rotor, a bend of a shaft, rigidity of housing, damaged ball bearing. These failures are included in our rotating machinery library for the diagnosis. Specifications of a motor, a coupling, a shaft, a rotor and bearings are decided at design process and set as parameters of the model. Each component shown in Figure 1 is built based on well-established rotor dynamics theory (Ishida Yamamoto, 2012; Matsushita et al, 2017).

The basic flange has 5 DOF (degree of freedom), consisting of 4 DOF (two dimensional deflection and slope) for transverse vibration of the rotor system and 1 DOF (angle) for torsional vibration, neglecting axial vibration. Figure 2 descripts the flange. The followings are 10 flange variables. 5 potential variables are

$u_{x}$ : Deflection of horizontal direction,

$u_{y}$ : Deflection of vertical direction,

$i_{x}$ : Deflection angle of horizontal direction,

$i_{y}$ : Deflection angle of vertical direction,

$\theta$ : Rotational angle.

Corresponding flow variables are
$F_{x}$ : Force of horizontal direction,

$F_{y}:$ Force of vertical direction,

$M_{x}$ : Moment of horizontal direction,

$M_{y}:$ Moment of vertical direction,

$T$ : Rotational Torque.

\subsection{Rotor}

In our library, the rotor is considered as a single mass in the form of a point mass, a rigid disc or a long rigid shaft. The rotor model is followed as 4 DOF Jeffcott rotor model. The forces and moments are given from next component through connector. The static unbalance model equations are following.

$$
\begin{aligned}
& m \ddot{u_{x}}-m e \dot{\theta}^{2} \cos \left(\theta+e_{0}\right)-m e \ddot{\theta} \sin \left(\theta+e_{0}\right) \\
& =F_{x} \\
& m \ddot{u_{y}}-m e \dot{\theta^{2}} \sin \left(\theta+e_{0}\right)+m e \ddot{\theta} \cos \left(\theta+e_{0}\right) \\
& +m g \\
& =F_{y}
\end{aligned}
$$

The dynamic unbalance model equations are following.

$$
\begin{aligned}
& I_{d} \ddot{i_{x}}+I_{p} \dot{\theta} \dot{i_{y}}-\left(I_{d}-I_{p}\right) \tau \dot{\theta^{2}} \cos \left(\theta+\tau_{0}\right) \\
& =M_{x} \\
& I_{d} \ddot{i_{y}}-I_{p} \dot{\theta} \dot{i_{x}}-\left(I_{d}-I_{p}\right) \tau \dot{\theta^{2}} \sin \left(\theta+\tau_{0}\right) \\
& =M_{y}
\end{aligned}
$$

Also, dynamic load torque effect is considered. 


$$
I_{p} \ddot{\theta}=F_{x} e \sin \left(\theta+e_{0}\right)-F_{y} e \cos \left(\theta+e_{0}\right)+T
$$

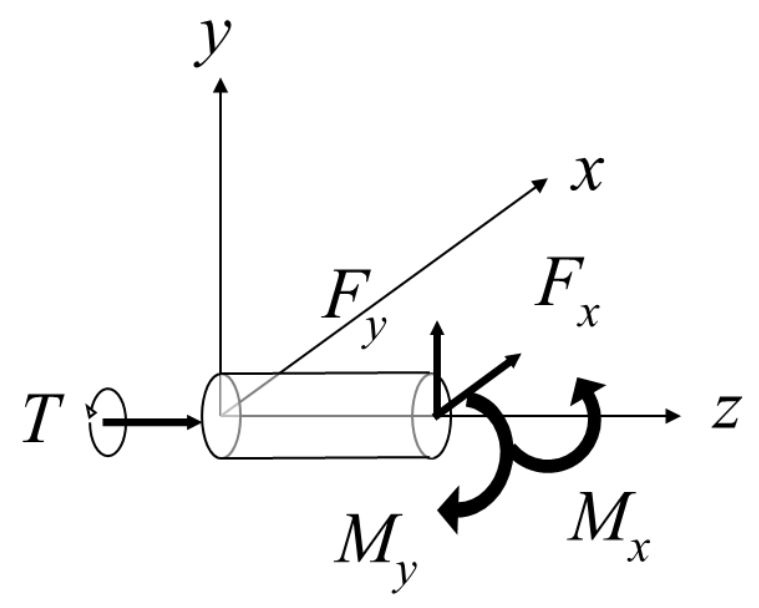

Figure 2. Flow variables of $f$ lange.

Here,

: Time-derivative operator (i.e. $\frac{d}{d t}$ ),

$m:$ Rotor mass,

$e:$ Eccentricity,

$e_{0}$ : Initial phase of eccentricity,

$\tau$ : Slope of dynamic unbalance,

$\tau_{0}:$ Initial phase of dynamic unbalance,

$I_{d}$ : Diametral moment of inertia,

$I_{p}:$ Polar moment of inertia.

\subsection{Shaft}

The shaft is considered as flexible (elastic) shaft. It is a linear elastic beam, uniform loading. The force and deflection relationships between two flanges follow.

$$
\begin{aligned}
E I\left(\begin{array}{l}
i_{b x} \\
i_{b y}
\end{array}\right) & =\frac{L^{2}}{2}\left(\begin{array}{l}
F_{a x} \\
F_{a y}
\end{array}\right)+L\left(\begin{array}{l}
M_{a x} \\
M_{a y}
\end{array}\right)+E I\left(\begin{array}{l}
i_{a x} \\
i_{a y}
\end{array}\right) \\
& +\left(\begin{array}{c}
0 \\
-\frac{m g L^{2}}{6}
\end{array}\right) \\
E I\left(\begin{array}{l}
u_{b x} \\
u_{b y}
\end{array}\right) & =\frac{L^{2}}{6}\left(\begin{array}{c}
F_{a x} \\
F_{a y}
\end{array}\right)+\frac{L}{2}\left(\begin{array}{c}
M_{a x} \\
M_{a y}
\end{array}\right)+E I L\left(\begin{array}{l}
i_{a x} \\
i_{a y}
\end{array}\right) \\
& +E I\left(\begin{array}{l}
u_{a x} \\
u_{a y}
\end{array}\right)+\left(\begin{array}{c}
0 \\
-\frac{m g L^{3}}{24}
\end{array}\right)
\end{aligned}
$$

Here,

$m$ : Shaft mass,

$L:$ Shaft length,

$E$ : Young's modulus,

$I$ : Second moment of area,

$g$ : Constant of gravitation,

Subscript $a$ is left flange, and $b$ is right flange.

\subsection{Bearing}

Bearing models have deflection rigidities in series and deflection angle rigidities in parallel. The bearing models also have damping factor. In addition to the above model, a bearing damage models on inner and outer ring are modeled by applying impulsive force. Bearing force relationships is following.

$$
\left(\begin{array}{l}
F_{x} \\
F_{y}
\end{array}\right)=k\left(\begin{array}{l}
\Delta u_{x} \\
\Delta u_{y}
\end{array}\right)+c\left(\begin{array}{l}
\frac{d \Delta u_{x}}{d t} \\
\frac{d \Delta u_{y}}{d t}
\end{array}\right)+F_{n} P\left(\begin{array}{c}
\cos \varphi \\
\sin \varphi
\end{array}\right)
$$

Here,

$k:$ Spring constant of bearing force,

$c$ : Damping constant of bearing force, $\Delta \boldsymbol{u}=\boldsymbol{u}_{\boldsymbol{c}}-\left(\boldsymbol{u}_{\boldsymbol{a}}+\boldsymbol{u}_{\boldsymbol{b}}\right) / 2$ : Difference of deflection, $F_{n}$ : Quantity of impulsive force due to ball and ring collision,

$P$ : If collision occurs, $P=1$, otherwise 0 ,

$\varphi$ : Impulsive force angle.

Subscript $c$ is housing or support flange.

We calculate the quantity of collision force using Hertz contact theory. Collision event is written by when statement.

Following is outer ring case.

$$
\begin{gathered}
F_{n}=1.143 m^{0.6} K^{0.4} v^{1.2} \\
v=\frac{D+d}{2} \omega_{1} \sin \theta_{d} \\
t=3.128 m^{0.4} K^{-0.4} v^{-0.2}
\end{gathered}
$$

Here,

$D$ : Diameter of inner ring,

$d$ : Diameter of ball,

$v:$ Ball collision speed,

$Z$ : Number of balls,

$\omega$ : Rotational speed.

$\omega_{1}$ : Ball orbital motion rotational speed,

$t:$ Contact time,

$\theta_{d}:$ Collision angle.

$K$ : Proportional constant of force.

$K$ is determined by elastic moduli and Poisson's ratio.

$$
\omega_{1}=\frac{D}{2(D+d)} \omega
$$

From relationships above, characteristic frequency of outer and inner ring collision events are described by ball orbital motion rotational speed.

$$
\begin{gathered}
f_{\text {outer }}=\frac{Z \omega_{1}}{2 \pi} \\
f_{\text {inner }}=\frac{Z\left(\omega-\omega_{1}\right)}{2 \pi}
\end{gathered}
$$

\subsection{Coupling}

Coupling models have both offset and angular misalignment. The deflections relationships between two flanges follow.

$$
\begin{gathered}
\boldsymbol{u}_{\boldsymbol{b}}-\boldsymbol{u}_{\boldsymbol{a}}=e\left(\cos \left(\theta+e_{0}\right) \sin \left(\theta+e_{0}\right)\right) \\
\boldsymbol{i}_{\boldsymbol{b}}-\boldsymbol{i}_{\boldsymbol{a}}=a\left(\cos \left(\theta+a_{0}\right) \sin \left(\theta+a_{0}\right)\right)
\end{gathered}
$$

Here,

$e:$ Offset misalignment length, 
$e_{0}$ : Initial phase of offset misalignment,

$a:$ Angular misalignment length,

$a_{0}$ : Initial phase of angular misalignment.

Coupling models also have deflection rigidities.

\subsection{Housing}

Housing model is spring damper against deflection. It dosen't have deflection angle rigidities.

\subsection{Support}

Support models have simple support, rigid support and free end. Simple support follows the condition that deflections and moments are zero. Rigid support follows the condition that deflections and deflection angles are zero. Free end follows the condition that forces and moments are zero.

\subsection{Motor}

Motor models are extended from Modelica.Electrical.Machines.BasicMachine $\mathrm{s}$ to be compatible with our library flange.

\section{Generation of data with fault}

Ball bearing in rotating machinery is very frequently damaged during operation. Inner and outer rings in bearing can be damaged due to over load, corrosion, improper lubrication and installation. Over load or weak lubrication causes friction. As a result of friction, temperature increases, so that oil lost its properties. Also, current flows on bearings, because of voltage difference between stator and rotor does. Oil acts as a dielectric material in condenser. These faults produce small particles in bearing.

Using our library, we generated outer and inner ring damaged ball bearing vibration case data in addition to static unbalance respectively. Figure 3 and 4 are simulation results respectively.

By building Modelica cyber system with the other faults refered in previous section, we can obtain time series data with some faults from Dymola simulation. Using these simulation generated data for training dataset after analysing and signal processing experimental measured data, it is possible making system fault detection algorithm based on machine learning theory (Figure 5). In case of the damaged ball bearing, envelope processing of bearing eigen frequency is required. Our final goal is generation of training data including unmeasurable quantities for diagnosis by statistical classification algorithms.

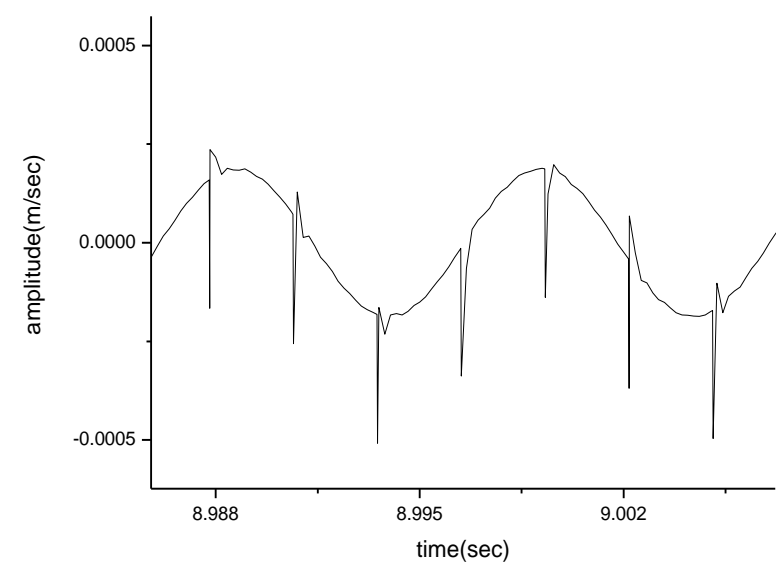

Figure 3. Outer ring fault with static unbalance.

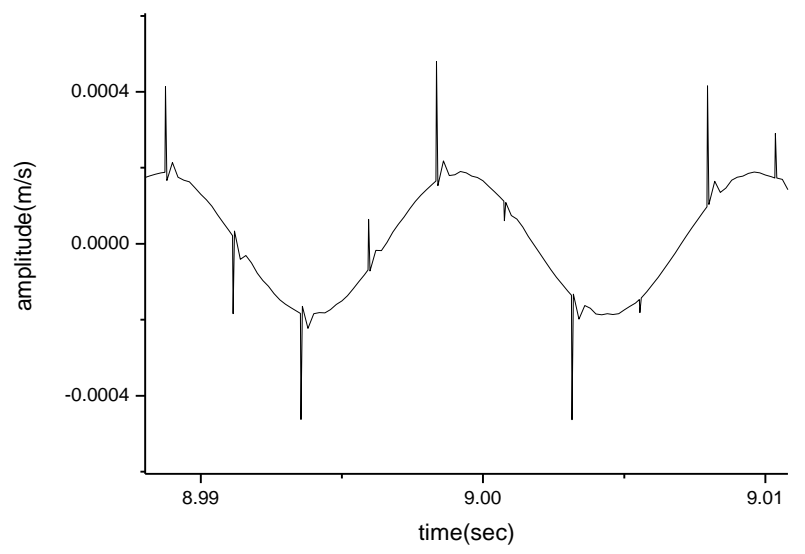

Figure 4. Inner ring fault with static unbalance.

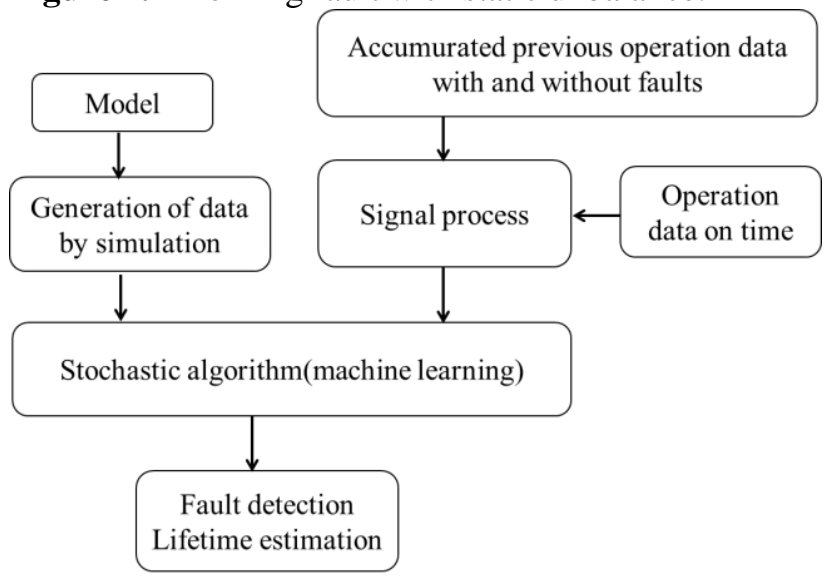

Figure 5. Diagnosis method using simulation data as training dataset.

\section{Model Validation}

\subsection{Experimental setup}

To validate our models, we built a rotor kit physical system shown in Figure 6. Vertical and horizontal deflection of a rotor were measured by two laser 
Table 1. Parameters of Modelica cyber system.

\begin{tabular}{|c|c|c|c|}
\hline \multicolumn{2}{|c|}{ Shaft } & \multicolumn{2}{|l|}{ Bearing } \\
\hline Length L [mm] & 500 & Width L2 [mm] & 15 \\
\hline Shaft diameter D1 [mm] & 6 & Damping constant [N. m. s/rad] & 0.053 \\
\hline length L1 [mm] & 50 & Rigidity [N. m/rad] & 23 \\
\hline Shaft diameter D2 [mm] & 8 & \multicolumn{2}{|l|}{ Coupling } \\
\hline Length L3 [mm] & 180 & Rigidity $[\mathrm{N} / \mathrm{m}]$ & 650 \\
\hline Length L5 [mm] & 50 & \multicolumn{2}{|l|}{ Motor } \\
\hline Young's modulus $[\mathrm{Pa}]$ & $2.06 \times 10^{9}$ & Torque [N. m] & 0.4 \\
\hline Density $\left[\mathrm{kg} / \mathrm{m}^{3}\right]$ & 8000 & Voltage [V] & 24 \\
\hline \multicolumn{2}{|c|}{ Rotor } & Rotating speed [rpm] & 2500 \\
\hline Diameter D4 [mm] & 80 & & \\
\hline Width L4 [mm] & 62 & & \\
\hline Young's modulus $[\mathrm{Pa}]$ & $2.06 \times 10^{9}$ & & \\
\hline Density $\left[\mathrm{kg} / \mathrm{m}^{3}\right]$ & 8000 & & \\
\hline
\end{tabular}

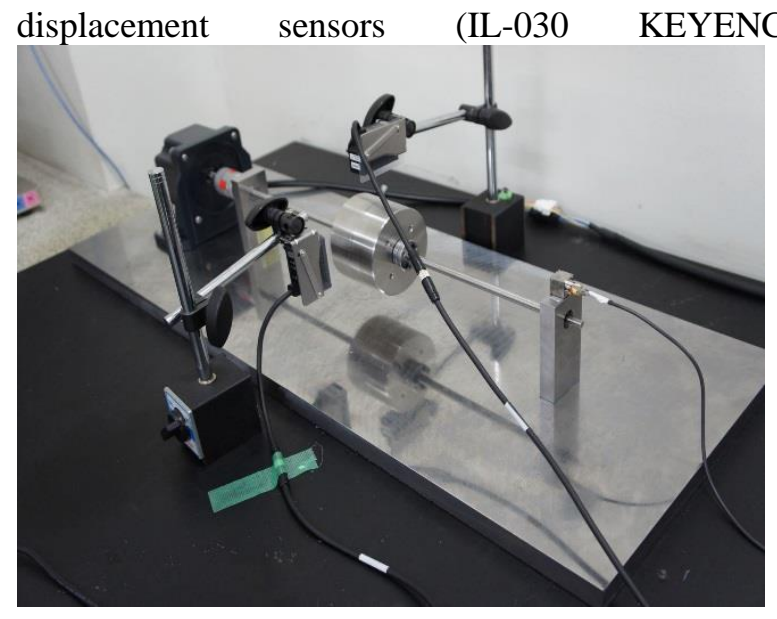

Figure 6. Rotor kit physical system.

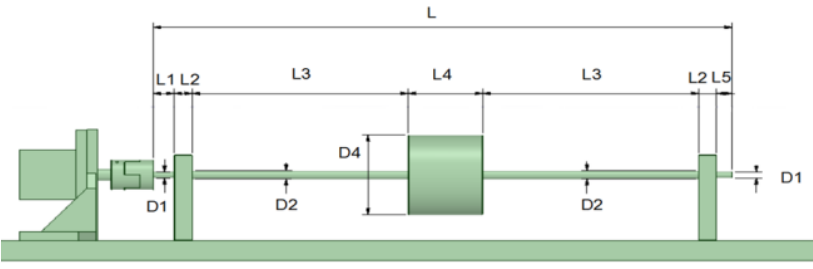

Figure 7. Parameters of Modelica cyber system.

We built Modelica cyber system based on our new library in Section 2 (Figure 1 and Figure 7). The parameters of Modelica cyber system are shown in Table 1. The parameters were determined by specifications of each component except bearing. The bearing support was neither simple support nor rigid support. It had some rigidity against the bending of the shaft. We determined the bearing model parameters of the deflection angle rigidity and damping from the preliminary impulse experiment.
To acquire data from the physical system to Dymola, we used National Instruments A/D and D/A Converter, Modelica_DeviceDrivers, Modelica_Synchronous and COMEDI (COntrol and MEasurement Device Interface for Linux / RTAI) (Ferretti et al, 2005). The OS was openSUSE Leap 42.1. The motor was AC Servomotor (NX410AA-1 Oriental motor). The input signal voltage driving motor was controlled through COMEDI. Figure 8 shows the rotor kit measurement and control system we built.

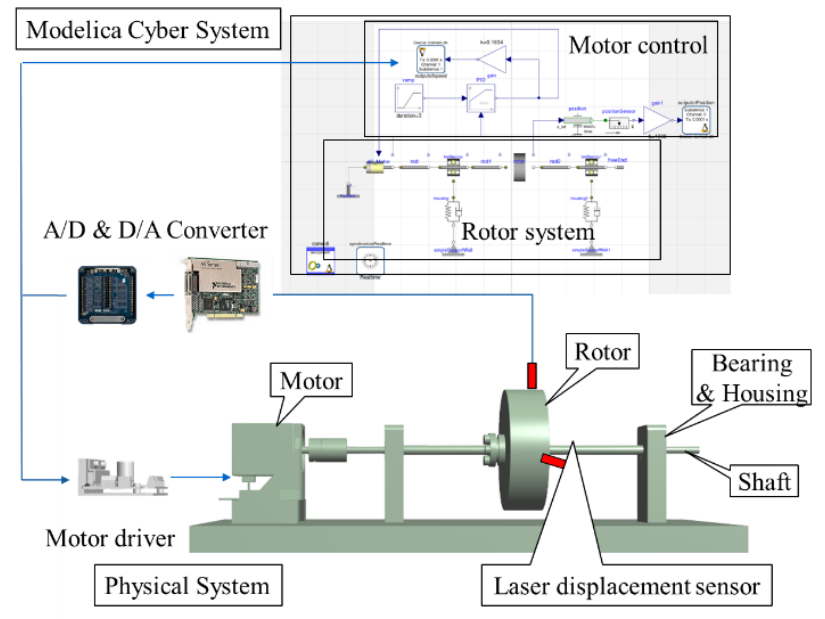

Figure 8. Rotor kit measurement and control system.

We tested the response of this system by applying step input to both physical system and Modelica cyber system. Figure 9 shows the response of motor speed and the rotor horizontal displacement vibration against the input voltage. A bit difference between experiment (physical system) and simulation (Modelica cyber system) was observed due to input signal overshooting in physical system. 


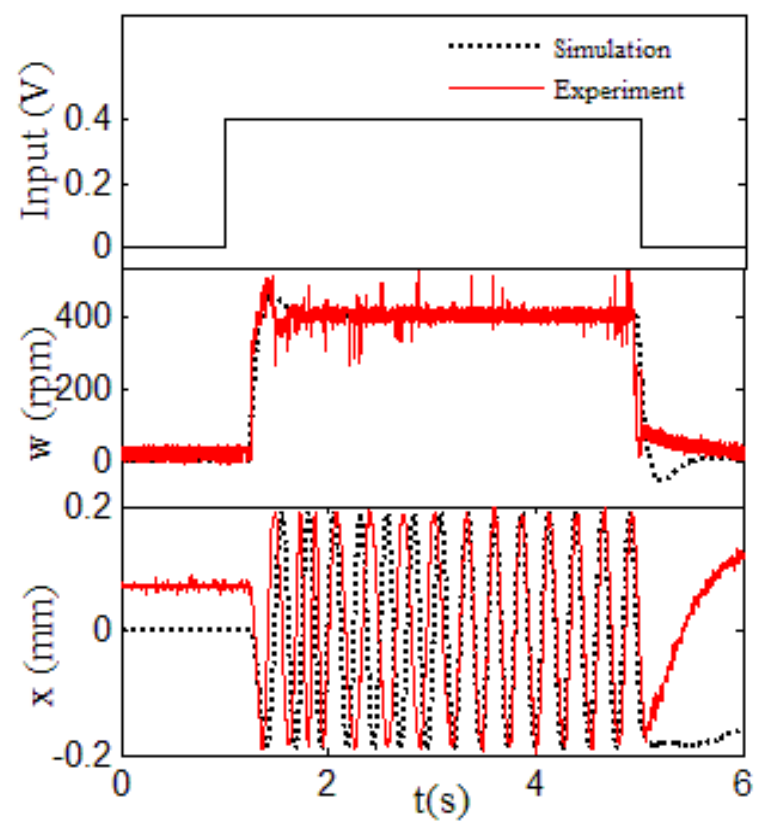

Figure 9. The step response of simulation and experiment. Input voltage, rotational speed and deflection versus time.

We also tested the ramp response of both systems. We rose the rotation speed up to $2500 \mathrm{rpm}$ over the first critical speed $1600 \mathrm{rpm}$. Figure 10 shows the result. The deflection beating over $1600 \mathrm{rpm}$ is due to equation (5) dynamic load torque effect of unbalance. By varying parameters of both eccentricity and bend of shaft, the responses of motor speed and horizontal vibration between simulation and experiment had little differences. By varying the bend of shaft value, we found the simulation became well-consistent with experiment.

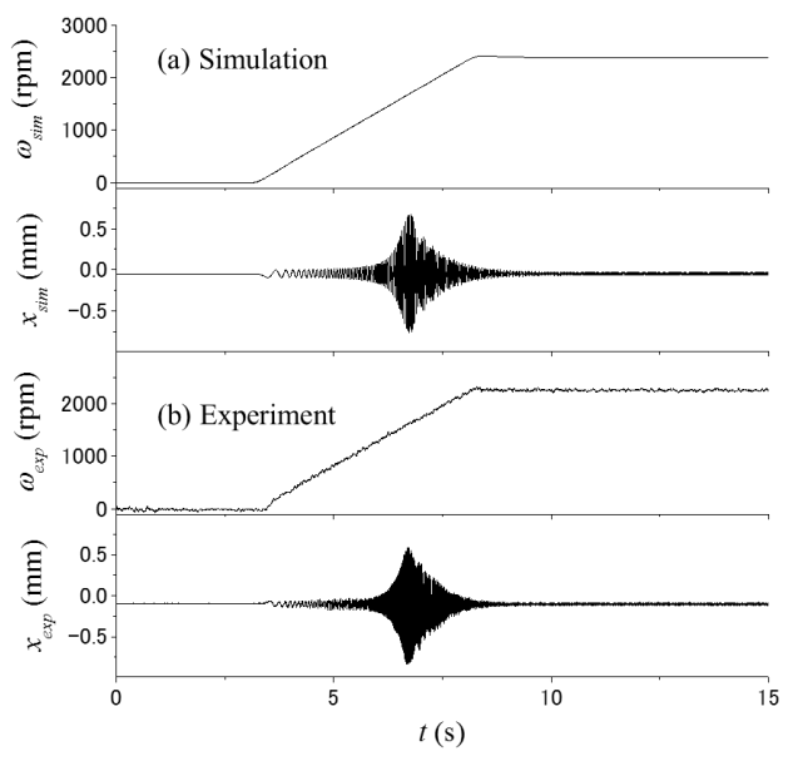

Figure 10. The ramp response of both system. Rotational speed and horizontal deflection versus time. (a) Simulation (Modelica cyber system). (b) Experiment (physical system).

\subsection{Correlation to Physical Test}

We calibrated the fault related parameter, as we found the bend of shaft had strongly correlated with the physical system. We preprocessed both simulation and experiment data by high pass filter at cutoff frequency $f_{c}=0.4 \mathrm{~Hz}$, envelope signal processing and low pass filter at cutoff frequency $f_{c}=10 \mathrm{~Hz}$. High pass filter was used to remove offset. Low pass filter was used to smooth the envelope curve. We optimized the bend of the shaft parameter by using Optimization Library (DLR, 2016). We used Integrated Squared Deviation as Criteria function and Pure Random Search algorithm for optimization.

$$
E=\int\left\{u_{1}(t)-u_{2}(t)\right\}^{2} d t
$$

Here,

$u_{1}(t)$ : Envelope processed simulation data.

$u_{2}(t)$ : Envelope processed experiment data.

Figure 11-13 show the result of the bend of the shaft optimization. The evaluation function dicreased during iteration (Figure 11). The envelope curve had changed after optimization (Figure 12). The bend of shaft value became $0.01 \mathrm{~mm}$, as initial value was zero. By setting this value as parameter for simulation model of Modelica, the simulation envelope curve became well consistent with experiment (Figure 13).

To validate the static unbalance model, we added a screw on the rotor and made static unbalance at the eccentricity $e=0.05 \mathrm{~mm}$. By setting the bend of the shaft parameter $0.01 \mathrm{~mm}$ and optimizing the eccentricity as above, the eccentricity parameter became $e=4.87 \times$ $10^{-2} \mathrm{~mm}$. We were able to estimate the eccentricity value from our library and Optimization library. Figure 14 shows the envelope curves of both the eccentricity parameter optimized simulation and experiment. We were able to generate the unbalanced rotor vibration data consistent with the physical system. Using Modelica cyber system, we can access the unmeasurable physical quantitities such as bearing load under operation. Simulating this kind of complex physical system is very hard by coding with Simulink. The more detailed simulation by the FEM (Finite Element Method) takes much time. Using Modelica, we can simulate and generate data very easily in a reasonable amount of calculation time 


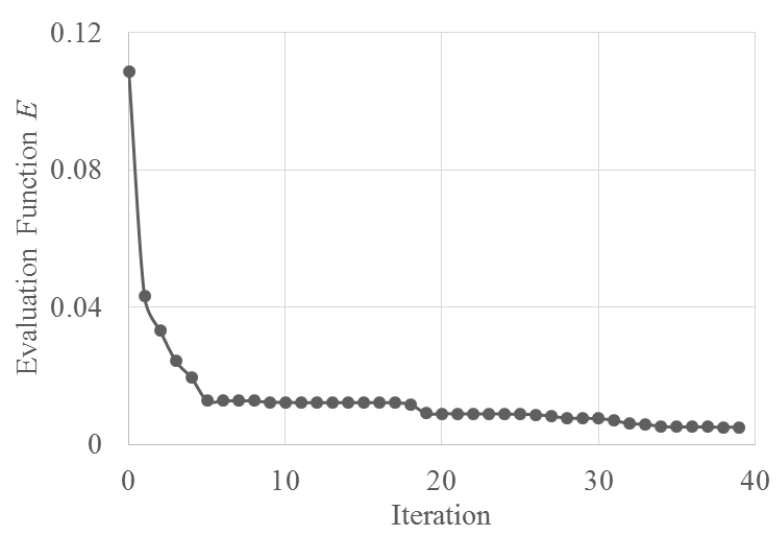

Figure 11. Evaluation function against optimization iteration.

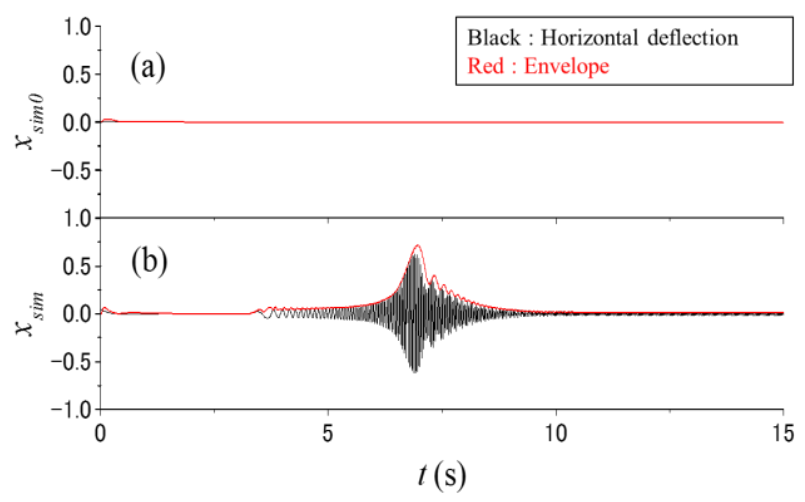

Figure 12. Envelope curve change by optimization. (a) Before optimization. (b) After optimization. Red line shows envelope of deflection signal.

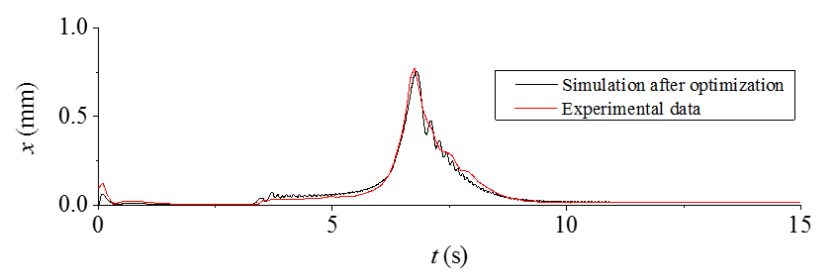

Figure 13. Comparison between simulation after the bend of the shaft optimization and experiment.

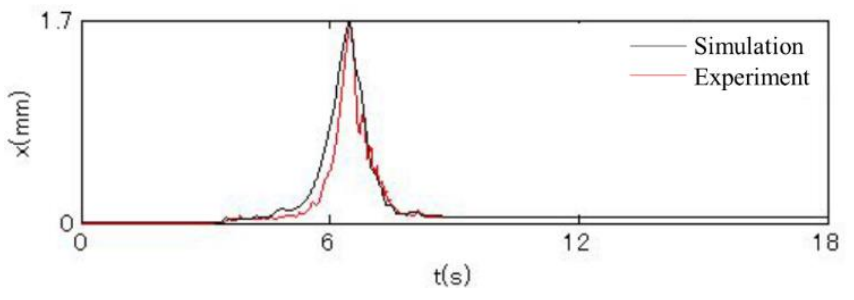

Figure 14. Comparison between the eccentricity optimized simulation (setting the optimized bend of the shaft parameter) and experiment with static unbalance.

\section{Conclusions}

This paper described Rotating Machinery Library we had developed based on rotor dynamics theory. This library generates rotating machinery system operation data with some faults. We validated our models by comparing both Modelica simulation and experiment with a rotor kit as a test case.

Further developments will focus on not only mechanical components, but also electrical components failures in rotating machinery system. Also, we will develop diagnosis methodology for identifying faults by stochastic and physical model based approach.

\section{Acknowledgements}

We gratefully thank Dr. Gao at Modelon KK, Japan for technical advices of developing library.

\section{References}

COMEDI Control and Measurement Interface http://www.comedi.org/

DLR, Optimization Library for Dymola Version 2.2.2 Tutorial, 2016.

Gianni Ferretti, Marco Gritti, Gianantonio Magnani, Gianpaolo Rizzi and Paolo Rocco. Real-Time Simulation of Modelica Models under Linux / RTAI. Proceedings of the 4th Modelica Conference 2005, pp. 359-365 Hamburg, Germany.

Yukio Ishida and Toshio Yamamoto, Linear and Nonlinear Rotordynamics: A Modern Treatment with Applications, 2nd Edition, Wiley-VCH, 2012.

Matthew Klenk, Johan de Kleer, Daniel G. Bobrow and Bill Janssen Using Modelica Models for Qualitative Reasoning. Proceedings of the 10th International Modelica Conference pp. 205-211, Lund, Sweden. doi: 10.3384/ecp14096205

Raj Minhas, Johan de Kleer, Ion Matei, Bhaskar Saha, Bill Janssen, Daniel G. Bobrow and Tolga Kurtoglu. Using Fault Augmented Modelica Models for Diagnostics. Proceedings of the 10th International Modelica Conference 2014, pp. 437-445, Lund, Sweden. doi: 10.3384/ecp14096437

Osami Matsushita, Masato Tanaka, Hiroshi Kanki, Masao Kobayashi and Patrick Keogh Vibrations of Rotating Machinery Springer Japan, 2017. doi: 10.1007/978-4-43155456-1 